\title{
Safe Prescribing: Vitamin D toxicity as a result of inadvertent overdose
}

\section{Dr. M Kurre, Dr. P Ramaswamy, Dr. E Pease-Gevers, Dr J Allgrove Royal London Hospital, Bart's Health NHS trust, London, UK}

\section{Introduction}

Vitamin D toxicity is rare; most of the reported cases were in adults and due to intake of high doses. In the last 5 years, there were few case reports of hypervitaminosis $D$ in children due to dosing or manufacturing error.

US Institute of Medicine produced guidance on tolerable upper intake level (UIL) in adults and children.

Serum levels of 25(OH)D above $500 \mathrm{nmol} / \mathrm{L}$ are deemed toxic.

NICE

Prevention dose- $\mathrm{RCPCH}$

Tolerable UIL

\begin{tabular}{|l|l|}
\hline Age & RNI \\
\hline $0-6 \mathrm{mo}$ & $340 \mathrm{IU}$ \\
\hline $6 \mathrm{mo}-3 \mathrm{yr}$ & $280 \mathrm{IU}$ \\
\hline$>65 \mathrm{yrs}$ & $400 \mathrm{IU}$ \\
\hline
\end{tabular}

\begin{tabular}{|l|l|}
\hline Age & \multicolumn{1}{|c|}{ Dose } \\
\hline $0-1 \mathrm{mo}$ & $\begin{array}{l}300-400 \mathrm{IU} \\
\text { daily }\end{array}$ \\
\hline $1-18 \mathrm{mo}$ & $\begin{array}{l}400-1000 \mathrm{IU} \\
\text { daily }\end{array}$ \\
\hline
\end{tabular}

\begin{tabular}{|l|l|}
\hline Age & Dose \\
\hline $0-6$ mo & 1000 IU \\
\hline $7-12$ mo & 1500 IU \\
\hline $1-3$ years & 2500 IU \\
\hline $4-8$ years & 3000 IU \\
\hline$\geq 9$ years & 4000 IU \\
\hline
\end{tabular}

\section{Naturopathy Products}

He was taking the below supplements.

Liqui-D3 2000 IU/drop, $1 \mathrm{ml}$ ( 30 drops) daily for 4 months

Cod Liver oil $5 \mathrm{ml}$ OD

Calcium Citrate 40mg TDS for 2 weeks

Purified Silver $50 \mu \mathrm{g}$ OD

Lipase Enzyme Complex 1400 units with meals

AFP digestive enzymes 1-6 caps per day

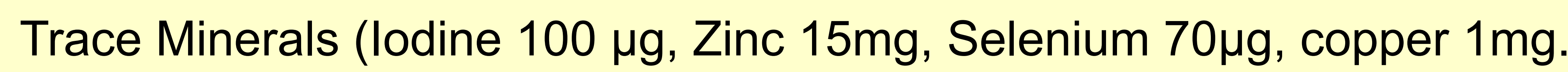

Mg $2 \mathrm{mg}$, chromium $200 \mu \mathrm{g}$, molybdenum $100 \mu \mathrm{g}$, boron $1 \mu \mathrm{g}$, vanadium

$250 \mu \mathrm{g}$, Silica $10 \mathrm{mg}$, Fat soluble Vitamin C 10mg)

Achturus Bromelain 500mg with meals

Sodium Chlorite Mineral solution 28\%

Marine salt $\mathrm{Hcl} 4 \%$

Camel milk 2cups a day

\section{Treatment}

\section{Dietary Ca restriction}

2.Hyperhydration $2.6-3.5 \mathrm{~L} / \mathrm{m}^{2}$ from day $3-8$ then $2.3 \mathrm{~L} / \mathrm{m}^{2}$ till day 12

3.Furosemide $1.5 \mathrm{mg} / \mathrm{kg} /$ day from day $2-8$, weaned over 2 days and stopped.

4.Calcitonin 40 units IM BD from day $2-4$, then IV $10 \mathrm{mg} / \mathrm{kg}$ on day 4,6 .

5.Pamidronate $0.5 \mathrm{mg} / \mathrm{kg}$ IV OD day $5-7$

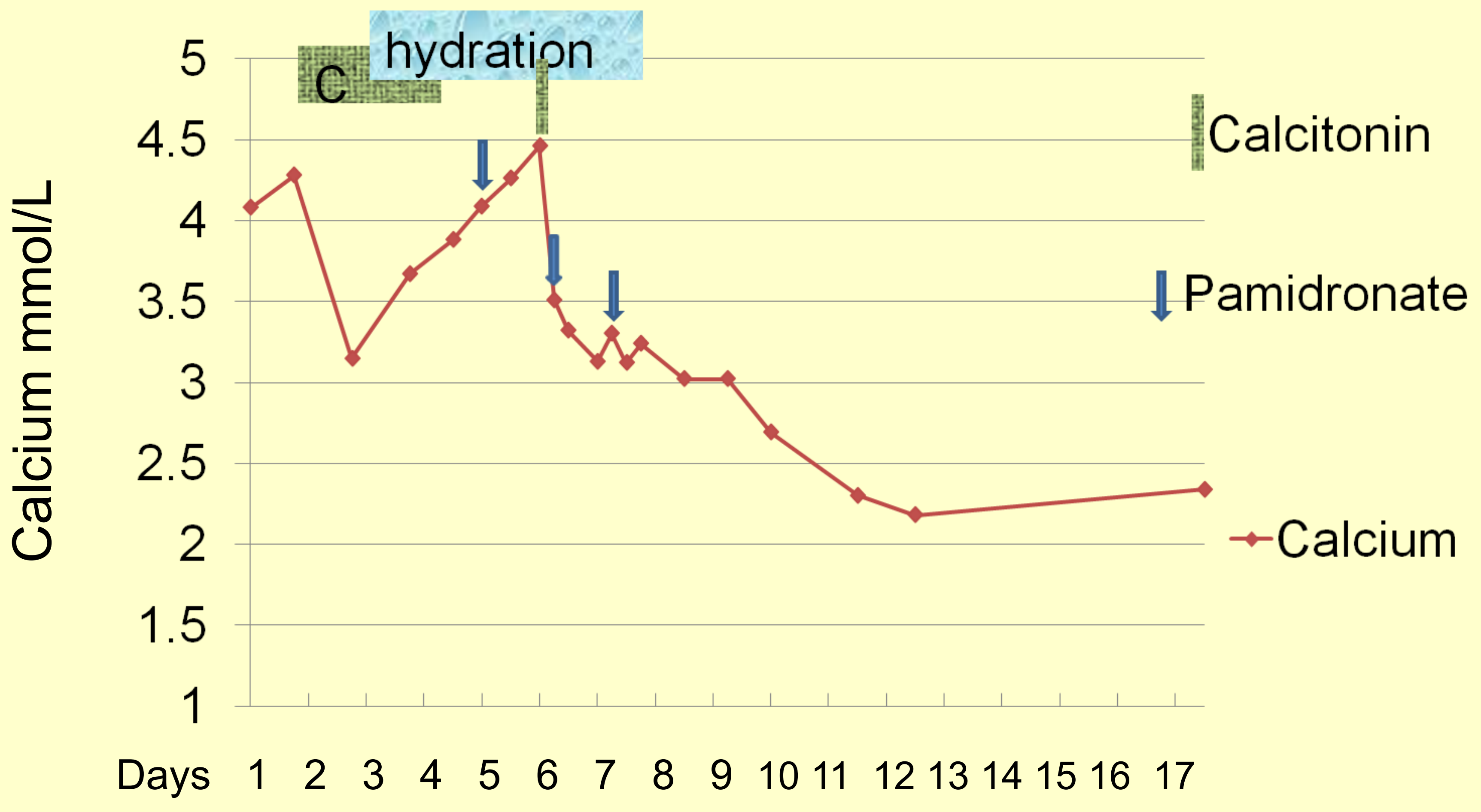

\section{References}

1. Vitamin D toxicity, policy and science; Reinhold Vieth; J Bone Miner Res 2007;22:S2;V64-V68.

. Institute of Medicine, Food and Nutrition Board. Dietary Reference Intakes for Calcium and Vitamin D. Washing ton, DC: National Academy Press, 2010.

25-Hydroxyvitamin D-24-hydroxylase (CYP24A1): Its important role in degradation of vitamin D; G. Jones et al. Archives of Biochemistry and Biophysics 523 (2012) 9-18

\section{Clinical Presentation}

4 year old child with back ground history of Autism presented with the following sequential symptoms

Constipation for 6-8 weeks

Loss of appetite for 4 weeks

Polydipsia of $1.5 \mathrm{~L}$ during the day for 3 weeks

Lethargy for 3 weeks

Vomiting for 2.5 weeks

\section{Investigations}

\begin{tabular}{|c|c|c|c|c|c|c|c|c|c|c|c|}
\hline & D1 & $\overline{\text { D2 }}$ & D3 & D4 & D5 & D6 & D7 & D7 & D10 & D12 & Range \\
\hline $\mathrm{mmol} / \mathrm{L}$ & 130 & 135 & 137 & 138 & 150 & 143 & 143 & & 140 & 149 & $133-146$ \\
\hline $\mathrm{mmol} / \mathrm{L}$ & 4.4 & 3.3 & 2.6 & 3.1 & 3.1 & 3.2 & 3.5 & & 3.4 & 5.2 & $3.5-5.3$ \\
\hline Urea $\mathrm{mmol} / \mathrm{L}$ & 9.7 & 4.6 & 3.2 & 3.4 & 5.6 & 4.7 & 5.0 & & 1.7 & 3.3 & $2.5-7.8$ \\
\hline Creat $\mu \mathrm{mol} / \mathrm{L}$ & 67 & 58 & 53 & 66 & 83 & 97 & 73 & & 32 & 31 & $27-42$ \\
\hline $\mathrm{mmol} / \mathrm{L}$ & 4.08 & 4.28 & 3.15 & 3.84 & 4.09 & 4.46 & 3.51 & 3.13 & 3.02 & 2.3 & $2.2-2.6$ \\
\hline PO4 $\mathrm{mmol} / \mathrm{L}$ & 1.1 & 1.0 & 0.7 & 1.0 & 1.1 & & 1.03 & 0.79 & 0.99 & 0.71 & $0.8-1.5$ \\
\hline unit/L & 145 & 137 & 133 & & 140 & & 109 & 94 & 97 & 92 & $55-350$ \\
\hline $\mathrm{mmol} / \mathrm{L}$ & 1.0 & 0.7 & 0.6 & & 0.5 & & & & 0.72 & 0.62 & $0.7-1.0$ \\
\hline PTH $\mathrm{pmol} / \mathrm{L}$ & & 0.6 & & & & & & & & & $1.6-6.9$ \\
\hline 25OHD nmol/l & & 2130 & & 1890 & & & 1154 & & & & $80-150$ \\
\hline \multicolumn{7}{|c|}{$1,25(\mathrm{OH})_{2} \mathrm{D} \quad \mathrm{pmol} / \mathrm{L}$} & 213 & & & & $<143$ \\
\hline U Ca $\mathrm{mmol} / \mathrm{L}$ & & & & 4.5 & & & & 1.4 & 1.3 & 1.1 & \\
\hline $\mathrm{U} \mathrm{Cr} \quad \mathrm{mmol} / \mathrm{L}$ & & & & & & & & 0.7 & 1.0 & 1.1 & \\
\hline
\end{tabular}

ECG - normal sinus rythm, QT interval

MRI Brain - No cerebral calcification

USS Kidneys - Mild Nephrocalcinosis

\section{Vitamin D Course}

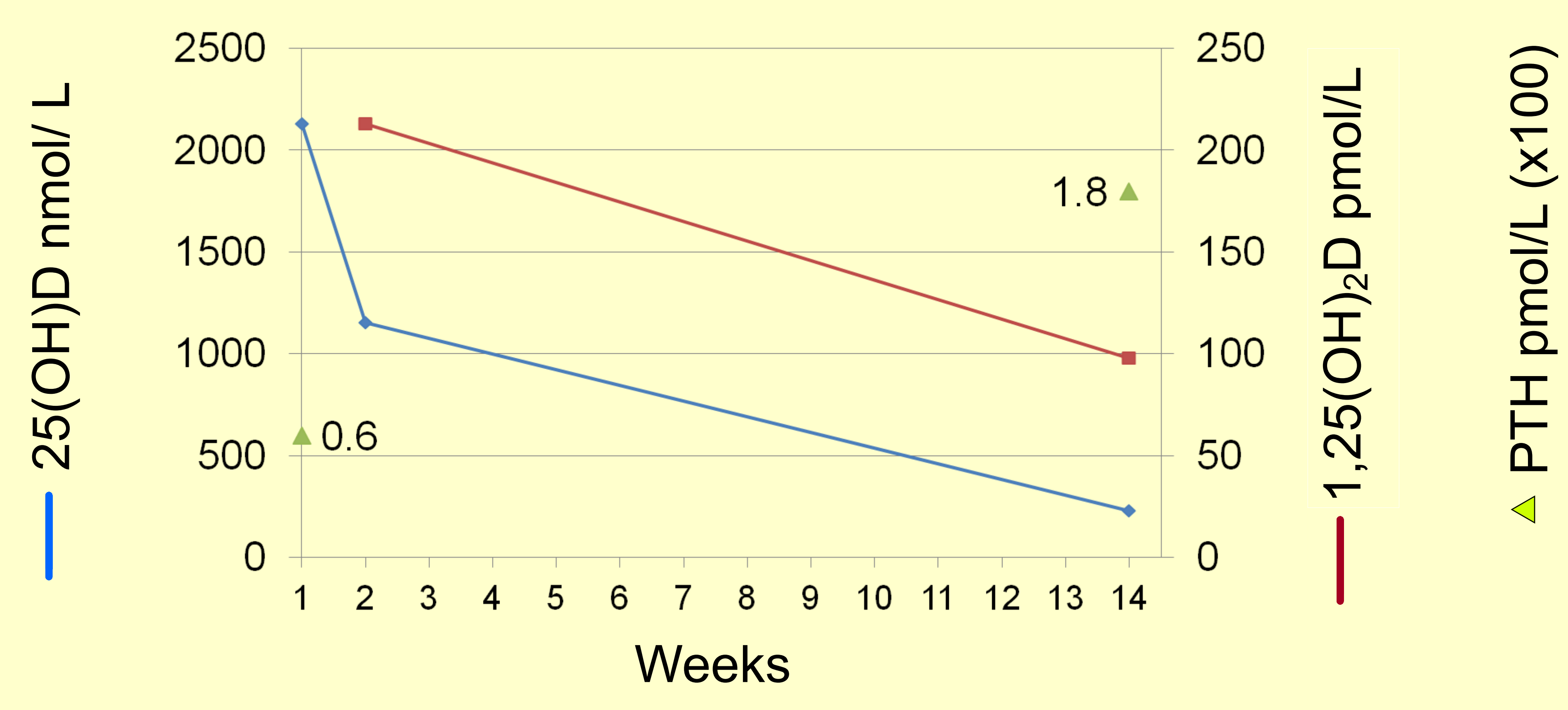

\section{Discussion}

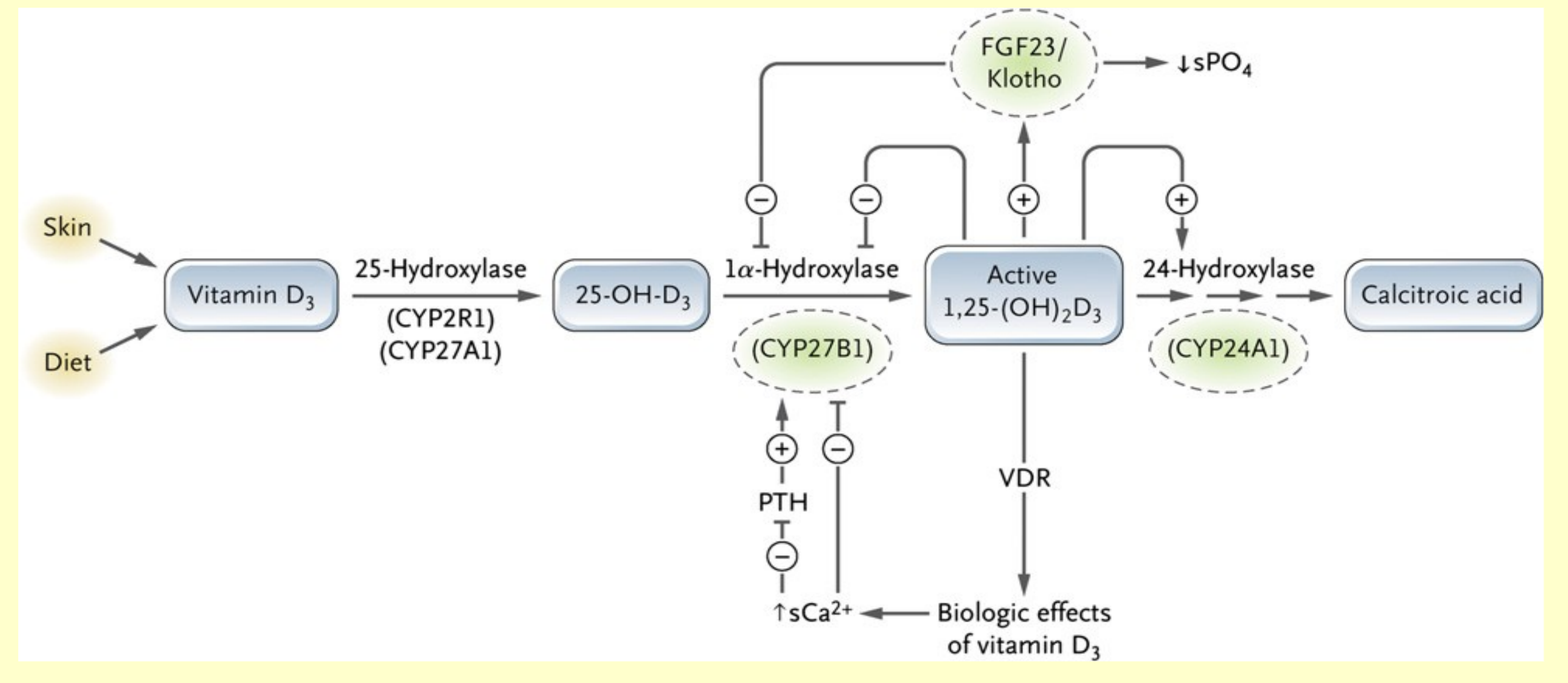

Plasma concentration of $1,25(\mathrm{OH})_{2} \mathrm{D}$ is regulated at a fairly constant level through both synthesis and catabolism.

As $25(\mathrm{OH}) \mathrm{D}$ level continues to rise, substrate driven output of $1,25(\mathrm{OH})_{2} \mathrm{D}$ is no longer regulated appropriately.

A differential diagnosis of 24 hydroxylase deficiency is being considered.

\section{Conclusion:}

We report a case of inadvertent overdose of concentrated Colecalciferol preparation, upto 60,000 IU per day, resulting in intoxication. Calcitonin brought reduction in calcium levels but there was rebound hypercalcaemia. Pamidronate infusion had sustained effect on regulating calcium levels. 